\title{
Density-functional study on the magnetic properties of Re atom adsorbed by $\mathrm{MoS}_{2}$ single layer
}

\author{
Zhao Hui Min ${ }^{1, a, *}$ \\ ${ }^{1}$ Bohai University, Jinzhou 121013, China \\ ahmzhao2008@163.com
}

\begin{abstract}
Keywords: $\mathrm{MoS}_{2}$ single layer, Re atom, Magnetic anisotropy
Abstract. We have investigated the magnetic properties of Re atom adsorbed by single layer $\mathrm{MoS}_{2}$ by first-principle calculation. Our results reveal that the cohesive energy of the adsorbed Re is $1.7 \mathrm{eV}$ and the total moment of the adsorbed system is $3.0 \mu_{B}$. The magnetic anisotropy reaches $19.0 \mathrm{meV}$ and the easy axis is perpendicular to the surface of $\mathrm{MoS}_{2}$ layer. The giant anisotropy mainly comes from the strong spin-orbital coupling of $5 \mathrm{~d}$ orbital of Re, which hybridizes with $2 \mathrm{p}$ orbital of $\mathrm{S}$.
\end{abstract}

\section{Introduction}

Low-dimensional materials have attracted much attention for their fascinating electric and magnetic properties. Previously, researchers believe that the strict single atom layer can not exist in real world due to the large thermal fluctuation in low-dimensional materials. However, this opinion has changed since the single layer graphene has been discovered and fabricated. Recently, metal-dichalcogenide $\mathrm{MoS}_{2}$ has attracted much attention due to their semiconducting properties. It has been widely used in lubricants, electrodes and catalyzers. Similar to graphene, the single layer $\mathrm{MoS}_{2}$ can be exfoliated from the bulk material.[1] The bulk $\mathrm{MoS}_{2}$ is the semiconductor with indirect energy gap of $1.29 \mathrm{eV}$, while the single layer is the semiconductor with direct energy gap of $1.8 \mathrm{eV}$. The single layer $\mathrm{MoS}_{2}$ has been used to fabricate the heterostructure of thin films, which have important application in optoelectronics and spintronics devices.[2] Pristine $\mathrm{MoS}_{2}$ is nonmagnetic and by doping or adsorbing the magnetic atoms, the magnetism can arise in the doped system. The magnetism of doped $\mathrm{MoS}_{2}$ has been investigated widely. However, the adsorption of transition metal atoms for $\mathrm{MoS}_{2}$ has rarely been studied.

In this article, we have investigated the magnetic properties of Re atom adsorbed by single layer $\mathrm{MoS}_{2}$ by first-principle calculation. The cohesive energy, density of states and energy band of the adsorbed system are calculated. We studied the effect of adsorption on the magnetic and electronic properties of the adsorbed system. Moreover, spin-orbital coupling is also included in our calculation and the magnetic anisotropy is calculated.

\section{Methods}

The lattice of MoS2 belongs to hexagonal system and the space group symmetry is P63/mmc. The distance between nearest Mo layers is $6.4 \AA$. The force of Van Der Waals type dominates between the layers and sliding is much easy to occur between layers. We build the single layer supercell with size of $3 \times 3 \times 1$ from the unit cell of $\mathrm{MoS}_{2}$. Totally, $9 \mathrm{Mo}$ atoms and $18 \mathrm{~S}$ atoms exist in the supercell. A slab of vacuum with thickness of $10 \AA$ was added to the layer to prevent the overlaps of atomic waves from the image layer. Re atom initially was relaxed from the position shifted above the surface of $\mathrm{MoS}_{2}$ with $3.0 \AA$ until it was adsorbed by three sulfur atoms. The relaxed structure was shown in Fig. 1 .

We calculate the magnetic properties of the adsorbed system by projected augmented wave method, as implemented into VASP computing code. The enegy cut off of plane waves is set to $400 \mathrm{eV}$. The conjugate-gradient algorithm is used to relax the atoms to equilibrium positions with the force criterion $0.01 \mathrm{eV} / \AA$. Generalized gradient approximation of Perdew-Burke-Ernzerhof form is used for 
the exchange-correlation potential. [3]Spin-orbital coupling was included via second variational method with scalar-relativistic basis.[4] A $7 \times 7 \times 1 \mathrm{k}$-mesh is used for the Brillouin sampling.

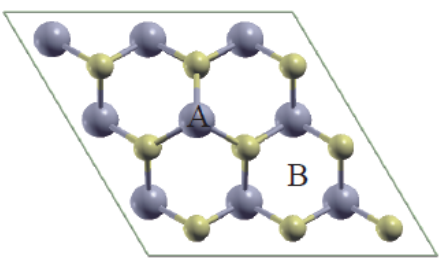

(a)

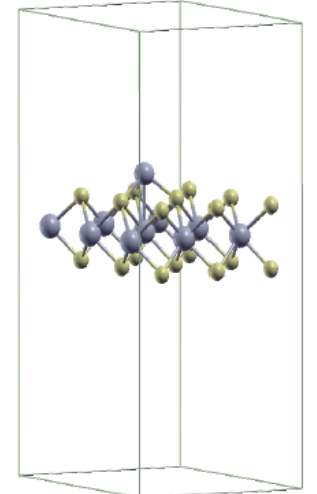

(b)

Fig. 1: (a) Top view of $\mathrm{MoS}_{2}$ layer adsorbed by Re. Possible sites of adsorption are labeled by A and B. (b) Side view of the adsorbed system. Re atom locates on the surface of sulfur layer. The yellow spheres represent sulfur atoms. Mo layer lies between the sulfur layers.

\section{Calculation results}

In the same layer, the sulfur atoms are arranged into the triangular lattice. Each Mo atom lies exactly below the center of three sulfur atoms. Therefore, there are two different positions for the adsorption of Re, as shown in Fig. 1(a). There is one Mo atom lies below the position of A while it is hollow for the position of B. Our calculations reveal that the system has lower energy when Re is adsorbed to the site A. The adsorption on A site is about $1.18 \mathrm{eV}$ lower in energy than the adsorption on B site. Therefore, we deduce that Re atom prefers to occupy the A site. Due to the adsorption, the three nearest $S$ atoms lift about $0.1 \AA$ compared to the other $S$ atoms. The bond length of Re-S is $2.22 \AA$, which is significantly less than the bond length of Mo-S. To indicate the magnitude of adsorption, we define the cohesive energy as $E_{c}=E(\mathrm{Re})+E\left(M o S_{2}\right)-E\left(\operatorname{Re} @ M o S_{2}\right)$, where $E(\mathrm{Re})$ and $E\left(M_{0} S_{2}\right)$ are the energy of isolated Re atom and pristine $\mathrm{MoS}_{2}$ layer. Our results reveal that the cohesive energy reaches $1.70 \mathrm{eV}$, which is larger than the cohesive energy of graphene adsorbed by magnetic atoms.

To study the electronic structure of the adsorption system, we calculate the density of states (DOS) and show them in Fig. 2. Due to the adsorption, local states are introduced into the energy gap. The local states mainly comes from the $5 \mathrm{~d}$ states of Re. Because of the $p$ - $d$ hybridization between $\mathrm{S}$ and $\mathrm{Re}$, additional states are introduced to the energy gap. Compared to the $5 \mathrm{~d}$ states of $\mathrm{Re}$, the $4 \mathrm{~d}$ states of Mo mainly lie below the Fermi level. Also, the spin splitting mainly occurs to the $5 \mathrm{~d}$ states of Re. For the majority states, two peaks emerge near the Fermi level, which is caused by crystal field. Our results reveal that Re has the local moment of $1.40 \mu_{B}$ while the nearest $\mathrm{S}$ atoms have the moment of $0.02 \mu_{B}$. The nearest Mo atom becomes also spin-polarized and has the moment of $0.45 \mu_{B}$. The total moment of the system is $3.0 \mu_{B}$. Bader charge analysis indicates that totally 1.1 charge transfers to the $\mathrm{MoS}_{2}$ layer.

We also calculate the spin-orbital coupling (SOC) of the adsorbed system. Essentially, SOC originates from the relativistic effect. The energy of SOC can be expressed as $E_{s o c}=-\langle\psi|\xi \hat{s} \cdot \hat{L}| \psi\rangle$, where $\xi$ is the parameter of SOC and $\xi \propto Z^{4}$. When heavy atoms are introduced and the system has much high DOS near the Fermi level, the SOC effect is remarkable. SOC can be affected by the spin orientation. Thus we define the magnetic anisotropy as $\Delta E=E_{s o c}(\|)-E_{s o c}(\perp)$, where $E_{s o c}(\|)$ and $E_{s o c}(\perp)$ are the energy of system with atom spin lying in and out of the plane respectively. Our results reveal that the magnetic anisotropy of Re atom is $19.0 \mathrm{meV}$ and the easy axis is out of the $\mathrm{MoS}_{2}$ plane. 
This value is significantly larger than the anisotropy of $9.0 \mathrm{meV}$ for single atom Co grown in $\mathrm{Pt}$ surface.[5]

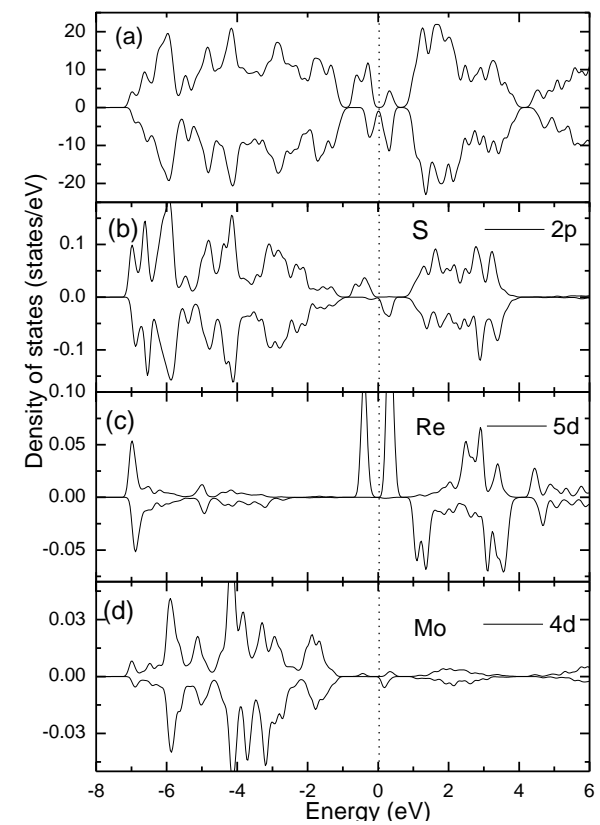

Fig. 2: (a) Total density of states. (b) 2p states of S. (c) 5 d states of Re. (d) $4 d$ states of Mo.

\section{Conclusions}

We have investigated the magnetic properties of $\mathrm{MoS}_{2}$ single layer adsorbed by Re atom. The cohesive energy is $1.70 \mathrm{eV}$ and the total moment is $3.0 \mu_{B}$. The adsorbed Re atom introduce local states in the energy gap. Our results reveal that the magnetic anisotropy is about $19.0 \mathrm{meV}$ and the easy magnetic axis is out of the $\mathrm{MoS}_{2}$ plane.

\section{Acknowledgment}

This work is supported by Social Science Foundation of Liaoning Province (No. L15DWW005) and Bohai University Teaching Reform Project (No.BDJG15QNB002).

\section{References}

[1] P. Joensen, R.F. Frindt, S.Roy Morrison, Single-layer $\mathrm{MoS}_{2}$, Mater. Res. Bull., 21 (1986)457-461

[2] B. Radisavljevic, A. Radenovic, J. Brivio, V. Giacometti, A. Kis, Single-layer $\mathrm{MoS}_{2}$ transistors,

Nat. Nanotech., 6 (2011) 147-150

[3] J. P. Perdew, K. Burke, and M. Ernzerhof, Generalized gradient approximation made simple,Phys. Rev. Lett. 77 (1996) 3865.

[4] A.D. Corso, A. M. Conte, Spin-orbita coupling with ultrasoft pseudopotentials: Application to Au and Pt. Phsy. Rev. B 71, 115106.

[5] P. Gambardella, S. Rusponi, M. Veronese, S. S. Dhesi, C. Grazioli, A. Dallmeyer, I. Cabria, R. Zeller, P.H. Dederichs, K. Kern, C. Carbone, H. Brune, Giant Magnetic Anisotropy of Single Cobalt Atoms and Nanoparticles,Science 300 (2003) 1130-1133 